\title{
Prácticas de paternidad de algunos varones gais de Ciudad de México. Entre tabúes y nuevas apuestas para su ejercicio ${ }^{1}$
}

\author{
Parenting Practices of Some Gay Men in \\ Mexico City. Between Taboos and New Bets \\ for their Practice
}

Práticas parentais de alguns homens gays na Cidade do México. Entre tabus e novas apostas para a sua prática

\section{Sebastián Giraldo Aguirre ${ }^{2}$}

Docente de la Universidad de Caldas, Manizales, Colombia

s.giraldoaguirre@gmail.com

Recibido: $22 / 09 / 14$

Aprobado: 14/04/15

\footnotetext{
1 Este artículo hace parte de la tesis "Padres en suspenso. Proceso de decisión, significados y prácticas de paternidad de algunos varones gay de la Ciudad de México", investigación realizada entre 2012 y 2014 para optar al título de maestría en Estudios de Género del Colegio de México.

2 Sociólogo. Magíster en Estudios de Género.
} 


\title{
Resumen
}

Aunque la paternidad de varones gais ha alcanzado protagonismo en los últimos años, aún se desconocen las maneras como ellos agencian su paternidad. El presente artículo esboza las prácticas de paternidad de cuatro hombres gais residentes en Ciudad de México, por medio de entrevistas y algunos documentos visuales recogidos en sus redes sociales de internet. Para ello se aborda el cuidado y el tiempo dedicado a los(as) hijos(as), y posteriormente, se expone un vínculo entre la autoridad, los valores transmitidos y la socialización de género de los menores. En general, se revela que sus arreglos parentales están en sintonía con las transformaciones de la paternidad contemporánea; sin embargo, deben reforzar algunos componentes debido a su orientación sexual.

Palabras clave: paternidad; masculinidad; diversidad sexual; familia.

\begin{abstract}
Although the paternity of gay men has achieved prominence in recent years, the ways they manage their paternity are unknown yet. This article outlines the parenting practices of four resident gay men that are inhabitants of Mexico City, through interviews and some visual documents collected in their social networking sites. Care and time spent with children are approached; then, the link between authority, transmitted values and gender socialization of children is exposed. In general, it is revealed that parental arrangements are in tune with the transformations of contemporary fatherhood; however, they should strengthen some components due to their sexual orientation.
\end{abstract}

Keywords: fatherhood; masculinity; sexual diversity; family.

\section{Resumo}

Embora a paternidade de varões gays tem conseguido protagonismo nos últimos anos, ainda se desconhecem as maneiras como eles agenciam sua paternidade. Este artigo descreve as práticas parentais de quatro varões gays residentes na Cidade do México, através de entrevistas e alguns documentos visuais coletados em suas redes sociais da internet. Portanto, aborda-se o cuidado e o tempo dedicado aos filhos, assim como o vínculo entre a autoridade, os valores transmitidos e a socialização de gênero. Em geral, se evidencia que os arranjos parentais são coerentes com as transformações da paternidade contemporânea; no entanto, devem reforçar-se alguns componentes relacionados com a orientação sexual.

Palavras-chave: paternidade; masculinidade; diversidade sexual; família.

Este trabajo está bajo la licencia Creative Commons Attribution 3.0

¿Cómo citar este artículo? / How to quote this article?

Giraldo, Sebastián. «Prácticas de paternidad de algunos varones gais de Ciudad de México. Entre tabúes y nuevas apuestas para su ejercicio». Sociedad y economía, n ${ }^{\circ} 29$ (julio - diciembre 2015): 39-62. 


\section{Introducción}

Las prácticas de paternidad hacen referencia a las labores desplegadas por los padres para el cuidado, la formación y el acompañamiento de sus hijos(as). Generalmente, la paternidad ha estado asociada con la autoridad y la provisión económica; sin embargo, distintos estudios realizados en Latinoamérica han indicado que las prácticas paternas registran algunas transformaciones tendientes a introducir relaciones más igualitarias y cercanas dentro de la familia (Fuller 2000; Viveros 2000; Olavarría 2000; Jiménez 2003). Entre estos cambios se encuentran: el auspicio de las manifestaciones de afecto, la participación en el cuidado de los(as) hijos(as), la promoción de la autonomía y el respeto por las decisiones de los menores.

Las prácticas de paternidad permiten formular marcos de análisis que integren los roles que actualmente cumplen los padres y cuáles son las particularidades cuando involucramos el tema de la orientación sexual. Vale la pena cuestionarse: ¿Será que la práctica de la paternidad de los varones gais es distinta? ¿Qué tanto influye su orientación sexual en la gestión de su rol paterno? ¿Qué particularidades se generan debido a su situación? De acuerdo con Bonino (2003), el estudio de la paternidad de varones gais resulta interesante porque permite observar lo que pasa con los hombres cuando las mujeres-madres no están presentes, indicio que genera otros interrogantes como: ¿A qué obedecen las divisiones en las tareas del cuidado de los(as) hijos(as) y de las labores del hogar? ¿Cómo se transforman las lógicas de socialización de los(as) hijos(as) que se ceñían a un marco heterosexual?

Según algunas investigaciones sobre paternidad gay en Latinoamérica (Herzberg 2005; Haces 2006; Libson 2012; Laguna 2013), la orientación sexual se convierte en un componente que termina interviniendo fundamentalmente en la práctica paterna, debido a que se constituye en un factor que obstaculiza o potencializa su puesta en escena. En este sentido, debe matizarse el supuesto de que el ejercicio paterno de varones gais no es muy distinto al de los demás padres, dado que la orientación sexual se convierte en una pauta negativa o positiva para la conformación de las prácticas paternas. Tampoco, podría catalogarse la paternidad de varones gais como completamente distinta, ya que en algunas ocasiones retoma patrones de otras paternidades para llevar a cabo su proyecto paterno. Un ejemplo de ello, como lo indica Haces (2006), es que los varones gais recibieron una socialización de género con todas las reglas o predisposiciones que este proceso implica; es decir, que adoptaron una formación genérica que, entre otras cosas, consolida un "deber ser" de paternidad que no integra la homosexualidad. De manera que dentro de la paternidad no heterosexual prevalecen ambivalencias difíciles de eludir.

Además de las transformaciones simbólicas y prácticas de la paternidad, y su relación con paternidades específicas, como el caso de la paternidad de varones gais, la emergencia de la diversidad sexual como un tema social y político es la otra premisa fundamental para el estudio de las paternidades no heterosexuales. Particularmente, en México D.F. en el 2010 se legisló a favor de la adopción por parte de parejas del mismo sexo, después de haber sido fallado el matrimonio igualitario unos meses atrás y las sociedades de convivencia en el 2007. Por 
otra parte, algunas cifras sobre la conformación familiar en México confirman la existencia de 250.000 familias nucleares gais en México; de esta cifra, 68,8\% tiene hijos, es decir, 172.000 familias, siendo más comunes las de parejas de mujeres ${ }^{3}$. Aunque estos números no precisan la prevalencia de las familias homoparentales compuestas por varones, son un indicio de la presencia de este tipo de formaciones familiares en el país.

Con los anteriores antecedentes, se puede comprender la pertinencia que tiene el estudio de la paternidad de varones gais en la actualidad, pues es un tema que reúne varias aristas para ser exploradas; además, es una oportunidad para desvirtuar algunas nociones que se tienen sobre este tipo de relaciones familiares. Más allá de ser un contenido de la agenda política de los grupos en defensa de la diversidad sexual, la paternidad gay de igual manera es materia de investigación con muchos terrenos desiertos que requieren ser abonados.

Por tal razón y con el fin de comprender la paternidad de los varones entrevistados desde su aspecto operativo, el presente artículo se divide en cuatro apartados. En el primero se detalla el acercamiento metodológico y en los demás se presentan los resultados de investigación. Inicialmente, se analiza la relación del tiempo dedicado a los(as) hijos(as) y el cuidado sobre ellos(as), haciendo alusión a las estrategias a las que acuden los padres para conciliar las divisiones en el cuidado de los menores y la dualidad trabajo/paternidad. Luego, se profundiza en la autoridad, la trasmisión de valores y la socialización de género asumida por los padres, tres tareas que van de la mano a la hora de asumir una educación moral sobre sus descendientes. Para terminar, se presentan algunas reflexiones finales.

\section{Metodología}

En el estudio participaron cuatro padres (Andrés, Ernesto, Fernando y Lorenzo) ${ }^{4}$ residentes en Ciudad de México y que tienen un perfil particular: reconocen públicamente su orientación sexual, todos viven con sus hijos(as), son personas activistas por la diversidad sexual, con niveles altos de escolaridad y de sectores medios. De manera que el análisis también involucra otras categorías como la clase, el nivel educativo y el perfil urbano, pese a que la orientación sexual es el principal elemento de investigación. La tabla 1 presenta alguna información sobre los padres.

El acercamiento a sus experiencias paternas se realizó por medio de entrevistas, encuentros que se supeditaron al testimonio de un solo padre, situación que compromete la riqueza interpretativa del objeto de estudio, ya que no se contó con los puntos de vista de otras personas que hacen parte del arreglo familiar. Los padres que aprobaron realizar el trabajo de campo dejaron en claro la condición de que en ningún momento se involucrara a sus hijos(as) dentro de

3 Información reunida por la Secretaría de Desarrollo Social (Sedesol), Consejo Nacional de Población (Conapo), Instituto Nacional de Estadística y Geografía (Inegi), Instituto de Investigaciones Sociales de la UNAM y el Colegio de México (2014).

4 A pesar que dos de los padres afirmaron que no tenían ningún problema en revelar sus nombres debido a su activismo político, se decidió asignarles pseudónimos a todos los entrevistados para buscar un trato igualitario. 
la investigación, argumentando que es una dimensión que hace parte de su vida privada, razón por la cual no se pudo plantear un acercamiento metodológico de tipo etnográfico. Tampoco se involucró a sus parejas, principalmente, para dar un manejo igualitario a todos los casos, pues dos de los padres no tenían pareja y los otros dos, sí. Por ello, la unidad de análisis del estudio se concentra en el padre entrevistado; su vida en pareja hace parte de la información contextual de su paternidad.

Tabla 1. Características generales de los padres entrevistados

\begin{tabular}{|l|l|l|l|l|l|}
$\begin{array}{c}\text { Pseudónimo } \\
\text { del padre }\end{array}$ & Edad & $\begin{array}{c}\text { Sexo y edad del } \\
\text { hijo }\end{array}$ & $\begin{array}{c}\text { Paternidad en } \\
\text { pareja o solo }\end{array}$ & $\begin{array}{c}\text { Forma como } \\
\text { fueron padres }\end{array}$ & \multicolumn{1}{|c|}{ Ocupación } \\
\hline Andrés & 45 & Hijo de 3 años & En pareja & Adopción & $\begin{array}{l}\text { Periodista y } \\
\text { consultor }\end{array}$ \\
\hline Ernesto & 57 & $\begin{array}{l}\text { Hijas de } 18,16 \text { y } \\
14 \text { años }\end{array}$ & Solo & Adopción & $\begin{array}{l}\text { Arquitecto } \\
\text { independiente }\end{array}$ \\
\hline Fernando & 47 & Hija de 2 años & En pareja & Adopción & Actor \\
\hline Lorenzo & 43 & Hija de 4 años & Solo & Hija biológica & $\begin{array}{l}\text { Sociólogo y } \\
\text { consultor }\end{array}$ \\
\hline
\end{tabular}

Fuente: elaboración propia.

Como fuentes de información secundarias, se recolectaron algunos documentos visuales, entre ellos, fotografías y videos publicados en redes sociales de internet por dos de los entrevistados; se revisaron notas periodísticas en las cuales los padres entrevistados son sus protagonistas, se exploró un blog realizado por uno de ellos en el que divulga varios escritos sobre su proceso de paternidad y se repasaron algunos estudios ya publicados en los que algunos de ellos también participaron.

Es necesario aclarar que la base principal de la reflexión son fuentes que están mediadas por la palabra del entrevistado, sobre lo que él representa de su paternidad, y no sobre datos obtenidos directamente por el investigador. Por esta razón, fue prudente establecer una distancia relativa respecto al discurso de los padres, que pudiera menguar la relación entre lo representado y lo actuado por el padre en sus prácticas de paternidad.

\section{Tiempo y cuidado, una encrucijada sin tantos azares}

El ejercicio de la paternidad involucra una serie de dimensiones que requieren ser detalladas por separado para así lograr un análisis más sistemático del tema. En palabras de García y de Oliveira, el ejercicio de la paternidad comprende distintas categorías entre las que se encuentran: la manutención económica, la relación de autoridad y los aspectos relacionados con la disciplina, el cuidado en lo que respecta a la alimentación, la higiene y la salud, la transmisión de conocimientos, y también el afecto, la comunicación y la cercanía entre padres e hijos(as) (2004, 283).

Teniendo de fondo lo anterior, en el presente estudio se retomaron algunas dimensiones mencionadas renglones atrás sobre el ejercicio paterno; sin embargo, fue necesario introducir otras nuevas ya que enriquecen el análisis de la 
paternidad del grupo de varones entrevistados. Las categorías retomadas fueron el tiempo dedicado a los(as) hijos(as), el cuidado y la autoridad, debido a que son elementos que resultan invariables en el rol paterno de la mayoría de los varones. Mientras que otros asuntos como los valores inculcados a los(as) hijos(as) y la socialización de género -que sí tienen una particularidad a partir de la orientación sexual de los padres-son los elementos inéditos de la reflexión. En el presente apartado se detalla la relación entre el tiempo y el cuidado dedicado a sus hijos(as).

El tiempo que se comparte con los(as) hijos(as) es una de las variables más complejas dentro del ejercicio paterno; por una parte, porque pugna con la dedicación laboral al que están impulsados los hombres y, por otra, debido a la distancia simbólica que se ha construido entre el padre y los(as) hijos(as) para preservar una figura autoritaria. Sin embargo, diferentes estudios (Torres 2002; Jiménez 2003; Salguero 2006; Rojas 2008) han demostrado que esta situación ha venido cambiando, sobre todo en sectores medios, pues parece que los varones cada vez están más interesados en estar presentes en la crianza de sus descendientes, lo que implica una mayor presencia en sus hogares. En el caso de la paternidad de varones gais, las cosas no son distintas, es más, se nota una preocupación más latente por estar acompañando a los(as) hijos(as), porque de lo contrario podrían ser señalados de forma negativa y así alimentarían los discursos en contra de su paternidad.

Mis prioridades se han diversificado y en esa diversificación entra mi hija, el poder ir con ella a clase de natación, o a veces que vaya mi esposo, yo voy a grabar. Este, dejé, por ejemplo, de dirigir novelas y preferí regresar a actuar (Fernando).

La llegada de los(as) pequeños(as) se convierte en un parteaguas en la vida de los varones, que regularmente buscan dejar atrás algunas cargas laborales y ciertos entretenimientos para así poder estar al lado de sus hijos(as). Los(as) hijos(as) se convierten en un turning point en la vida de los varones, no solo por la satisfacción personal y la responsabilidad económica que requieren, sino también por el tiempo que demandan para su crianza.

Los(as) hijos(as), además de intervenir en la agenda personal de cada padre, inciden en los tiempos y actividades de la pareja. Algunos entrevistados comentaron que los planes con su compañero sufrieron un reacomodo a partir de la llegada de los menores pues, entre otras cosas, tuvieron que sacrificar fiestas y viajes que regularmente hacían juntos. Aun así, estos cambios nunca son vistos como un sacrificio, todo lo contrario, es una fuente de satisfacción para los padres, pues no les importa dejar de lado algunos pasatiempos por estar atentos en la crianza de sus hijos(as).

Generalmente, la cantidad de tiempo es mayor cuando los(as) hijos(as) están pequeños(as) y con el pasar de los meses o años, los periodos de dedicación exclusiva se van acortando. $\mathrm{Al}$ respecto Andrés y Lorenzo comentan:

Los primeros días fueron muy hermosos, mi esposo y yo totalmente dedicados única y exclusivamente al niño (Andrés). 


\begin{abstract}
Los primeros meses obviamente trabajaba menos y estaba más con ella, porque, por ejemplo, yo no salía todo en el día, solo en la tarde, y eso si era necesario; si no hacía todo por casa, o si estaba en un taller los daba en la tarde, para estar toda la mañana con ella (Lorenzo).
\end{abstract}

Pese a que los períodos de dedicación se van reduciendo al aumentar la edad, por lo menos durante los primeros cinco años de vida o básicamente hasta la entrada a la escuela primaria, los padres entrevistados mantuvieron cierta constancia en flexibilizar sus horarios de trabajo para poder compartir más actividades con sus pequeños(as). Recordemos que los(as) hijos(as) de tres entrevistados todavía se encuentran en etapas preescolares. Sobre el tema, Ernesto, quien es el único que tiene hijas adolescentes, hizo referencia a que las cosas eran distintas cuando las niñas eran más pequeñas, haciendo alusión al tiempo que ellas requerían.

Por otra parte, existen parámetros de la distribución de roles de género que, aun en situaciones homoparentales, inciden en la capacidad del tiempo que los padres pueden pasar con sus hijos, siendo uno de ellos la relación trabajo/ masculinidad. Los hombres son llamados a estar inmersos en el mundo laboral de una manera más contundente que las mujeres, situación que limita otras dimensiones de su vida, entre ellas la paternidad. Sin embargo, lo anterior no se convierte en un determinante fundamental de las relaciones paternas; los varones buscan estrategias para conseguir estar más tiempo en casa.

\footnotetext{
Yo procuro estar más tiempo con ella, digo, la ventaja que yo tengo es que en mi trabajo yo soy el que determina los horarios, entonces me doy mis tiempos para estar con ella; excepto cuando salgo, pues obviamente ella se queda en la casa (Lorenzo).

Evito citas, evito cuestiones del trabajo en la tarde para estar más tiempo con ellas, pero eso me ha repercutido en la agenda, con las distancias de la ciudad es muy complicado. Tengo la fortuna de trabajar independiente, entonces busco darles mis tiempos (Ernesto).
}

Los padres entrevistados tuvieron la suerte de contar con recursos económicos que permitieron adaptar sus obligaciones laborales, todos ellos se encontraban en empleos flexibles o independientes en los que no tenían un horario fijo. Gracias a esa situación, pudieron organizar mejor su agenda con el fin de estar más tiempo con sus hijos(as). Y no solo tuvo que ver con su estado laboral; algunos de ellos explicaron que tenían ahorros que les permitieron renunciar a su trabajo o desechar ofertas laborales, sobre todo, durante los primeros meses de vida de los(as) pequeños(as); realidad que fue cambiando cuando sus hijos(as) fueron creciendo, pues ya empezaron a buscar otros horizontes financieros, eso sí, que no dejaran de contemplar la posibilidad de estar el mayor tiempo posible al lado de ellos(as).

La armonía entre el trabajo y la paternidad es un asunto difícil de conciliar. En su estudio, Torres (2002) señala que uno de los obstáculos principales que los varones observan para ejercer su paternidad es el tiempo que disponen para la crianza, debido a su trabajo. En este sentido, como lo declaran algunos estudios (Barker y Verani 2008; Mena y Torres 2013), existe una clara conexión entre el empleo de los hombres y su participación como padres. En el caso de los 
entrevistados, la flexibilidad laboral en la que están inmersos, unos por sus trabajos independientes y otros por la cualidad de su ocupación, les ha permitido estar presentes mayor tiempo en la crianza de los(as) hijos(as). La búsqueda de estructuras laborales sin horarios fijos ni altas responsabilidades es una de las características comunes de los padres abordados, situación que no es distinta a la de otros estudios sobre paternidad de varones gais como el de Haces (2006). Incluso no es un asunto exclusivo de este tipo de paternidad, pues estudios como el de Gutmann (2000) también lo han referido sobre paternidades heterosexuales y de otros sectores sociales ${ }^{5}$.

Los arreglos en la agenda llevados a cabo por los padres igualmente sacan a flote la preocupación de los varones por la proveeduría económica de los(as) hijos(as). La paternidad generalmente se ha asociado con la provisión monetaria de la familia -muchos estudios de la región lo han considerado así (Olavarría 2000; García y Oliveira 2004; Rojas 2008)- y pese a que estemos en un período de transformación del rol paterno en el que emergen otros tipos de paternidad como la gay, este aspecto todavía es primordial en las representaciones genéricas de los varones. Sin embargo, Figueroa y Franzoni (2011) sostienen que se ha venido generando un cambio de valores en el que el sentimiento y la responsabilidad moral sobre los(as) hijos(as) han venido desplazando el interés económico de los padres, por tal motivo el éxito monetario y la búsqueda de reconocimiento social, que obliga a los hombres a destinar más horas al trabajo, va cambiando por el mayor tiempo destinado al cuidado de los menores $(2011,8)$.

Para muchos hombres ser padre implica inmediatamente una responsabilidad económica, idea de la que no se escapan los varones entrevistados, pues en algunas líneas de sus testimonios delatan su preocupación por la provisión de sus descendientes ${ }^{6}$. Por más que exista un dilema entre su trabajo y su paternidad, se procura estar el mayor tiempo posible con los(as) hijos(as), sobre todo en las labores de aseo, alimentación y a la hora de despertar o acostar a los(as) pequeños(as). Fernando comenta al respecto: "Nosotros procuramos estar siempre en lo más importante, en la bañada, en los teteros, dárselos nosotros y así fue desde que llegó, y desde el principio hemos estado con ella, afortunadamente yo dejé de trabajar los primeros meses".

Para los padres es una satisfacción y una razón de tranquilidad estar en las actividades que ellos consideran fundamentales, debido a que es un indicador de la buena crianza que le están intentando brindar a sus hijos(as). De manera que el tiempo que se comparte en familia también es un recurso que brinda bienestar a los padres, y no solo a los(as) hijos(as) como podría pensarse. Para los varones entrevistados, estar al lado de sus hijos(as) es una de las mayores gratificaciones que pueden tener durante el día después de haber tenido una jornada

5 En su estudio sobre masculinidad en Santo Domingo, barrio popular del sur de México D.F., el autor sostiene que los varones prefieren empleos en los cuales puedan estar pendientes de sus hijos(as), por eso buscan posibilidades laborales dentro de la vecindad.

$6 \mathrm{Al}$ respecto, Figueroa y Flores (2012) sostienen que para los varones es más sencillo incorporar la cercanía y el cuidado con sus familiares, sobre todo con sus hijos(as), cuando ello no implica una renuncia total a sus responsabilidades económicas como proveedores $(2012,50)$. En este sentido, como lo señalan Mena y Torres (2013), la proveeduría económica se presenta como un elemento que presenta grandes resistencias a modificarse en el ejercicio de la paternidad. 
extenuante. Por eso, los períodos que se planean para estar con los menores no son vistos como una carga, sino como momentos de placidez. Además, el tiempo que se comparte con ellos no se limita a la casa; a la vez implica otros espacios en los que los padres de alguna forma se distraen de su rutina laboral. Algunos de ellos están en clases de natación con sus hijos(as), en cursos de música o en otras actividades en las que participan de forma directa.

Distintos autores han señalado que una participación activa de los padres en la crianza de sus hijos(as) es fuente de bienestar y satisfacción en la vida de los varones (Cebotarev 2003; Nudler y Romaniuk 2005; Barker y Verani 2008; Echeverría 2012) ${ }^{7}$. Esta premisa es comprobada con los cuatro padres entrevistados, pues sus testimonios y sus gestos durante las entrevistas dan cuenta del grado de felicidad que les generan sus hijos(as), sentimiento que se condensa principalmente cuando comparten actividades de cuidado y recreación con ellos(as). En general, la decisión de estar más presentes en la crianza de sus descendientes proviene de una disposición personal de los padres. Sin embargo, en dos casos en particular, los que son padres adoptivos, hubo un refuerzo por parte de las instituciones (Desarrollo Integral de la Familia y Procuraduría) para que estuvieran el mayor tiempo posible con los(as) pequeños(as) los primeros años de vida $^{8}$. Este precepto estaba muy presente en Andrés y Fernando y trataban de realizarlo.

De una forma u otra, la división de los tiempos obedece a una jerarquía de actividades que determinan la selección de unas y el descarte de otras para poder coordinar la responsabilidad laboral con el ejercicio paterno, dilema que se vuelve más sencillo de concertar gracias a la flexibilidad laboral de los cuatro padres y a la ayuda que la prestan familiares y nanas en el cuidado de los(as) hijos(as). Así entonces, el tiempo dedicado a los menores tiene una relación directa con el cuidado hacia ellos, pues son variables dependientes que repercuten en la relación padre/hijo(a) de manera transversal, aunque no se puede caer en la trampa de pensar que la calidad de la participación del padre es proporcional al tiempo que pasa con los(as) hijos(as), sino que es una relación con más aristas.

Pasando al tema del cuidado, se debe tener en cuenta que es un asunto que gana centralidad respecto a otras dimensiones de la práctica paterna, más cuando estamos haciendo referencia a varones gais, debido a dos circunstancias en especial: primero, porque son arreglos familiares que no se acomodan a una clara división de roles tradicionales; y segundo, debido a que muchos varones no cuentan con las habilidades para el cuidado de menores debido a la socialización de género recibida. Sobre el último punto, algunos padres entrevistados

7 Argumento que también ha sido sustentado por Juan Guillermo Figueroa en el texto "Paternidad, mortalidad y salud: ¿es posible combinar estos términos?" (Figueroa 2011a), aludiendo que debido a los modelos dominantes de masculinidad, muchos padres se privan de experiencias potencialmente gratificantes y lúdicas que podrían ser reforzadoras de su salud física y emocional.

8 El Desarrollo Integral de la Familia (DIF) y la Procuraduría son las instituciones que están a cargo del sistema de adopción en México. Los dos padres declararon que hubo varias recomendaciones, sobre todo, impartidas por los despachos de psicología. Una de las sugerencias era estar el mayor tiempo posible con los(as) pequeños(as), según ellos, porque los(as) niños(as) adoptados(as) requieren más acompañamiento para evitar posibles consecuencias emocionales en su desarrollo psicosocial. 
comentan que no tenían las destrezas suficientes para ciertas actividades referidas al aseo, la alimentación y el sueño de los(as) pequeños(as).

En el caso de los varones que comparten la paternidad con su pareja, el tema de las divisiones de actividades no obedece a un patrón definido y permanente, sino que lo hacen por destrezas y por el tiempo que tienen uno u otro'. Este tema fue aludido por Haces (2006) en su estudio, quien incluso señala que este tipo de acuerdos demuestran que estos padres logran flexibilizar sus roles genéricos y se permiten hacer labores que dentro de las figuras parentales tradicionales no coinciden con el papel de padre. Se debe agregar que los grados de participación en el cuidado y la crianza de los(as) hijos(as), como apunta Mena (2009; 2013), están influidos por otros factores como: la actitud de los padres, el tiempo que poseen, la forma en que fueron educados y nivel de escolaridad; otros autores (García y de Oliveira 2004; Barker y Verani 2008) agregan otros componentes como los antecedentes urbanos y la edad del padre ${ }^{10}$.

Los varones entrevistados acudieron a distintas estrategias para el cuidado de sus hijos(as), en las cuales confluye un cuidado exclusivo por parte de ellos durante algunos horarios del día y algunas ayudas, principalmente femeninas, en manos de familiares o nanas. Cuando los(as) pequeños(as) entran a la escuela, las cosas cambian un poco. Como se explicó renglones atrás, el cuidado de los menores requiere mayor atención en los primeros meses de edad y con el paso del tiempo se va construyendo otro tipo de arreglos de pareja y familiares para atender a los(as) pequeños(as). Andrés dice: "Mi mamá nos ayudó dos o tres días. Un amigo que es enfermero también nos apoyó como tres, cuatro noches, junto con mi mamá, las noches nada más, y de ahí adelante éramos mi esposo y yo".

Durante los primeros días de la llegada de los(as) hijos(as), los padres tuvieron una dedicación casi exclusiva a los menores, se ausentaron parcialmente de sus responsabilidades laborales y acudieron a algunos ahorros para poder suplir el cuidado que demandaban sus hijos(as). Algunos entrevistados comentan que tuvieron que acudir a familiares cercanos, casi siempre representado por las abuelas o nanas para asistir las atenciones de los infantes; según ellos, porque no sabían muy bien cómo se cambiaba un pañal, cómo se bañaba un bebé o no tenían los suficientes conocimientos para atender los llantos de los(as) pequeños(as):

\footnotetext{
La beba se levanta con nosotros, desayuna con nosotros, yo he estado grabando mucho, cuando yo voy a grabar se queda mi esposo o la nana, cuando mi esposo se va también a hacer sus cosas, se queda con la nana, yo regreso a veces también a jugar con ella cuando tengo tiempo (Fernando).
}

Durante toda la mañana el niño está con nosotros, ya sea conmigo o con mi esposo solo, o con los dos (Andrés).

9 Lo mismo es señalado por Donoso (2002) para el caso de familias homoparentales compuestas por mujeres en España. En específico, sostiene que debido a que no hay patrones prescritos de género, las madres establecen roles negociados que siguen principios de distribución que responden a habilidades, gustos e intereses, como a las circunstancias particulares de cada familia. En este punto se debe recordar que los padres entrevistados tienen un nivel educativo alto, que su paternidad es resultado de un proceso de reflexión profundo, que tienen edades entre los 40 y 60 años, que viven en zonas urbanas y que están inmersos en estructuras laborales flexibles. 
Generalmente el cuidado exclusivo de los padres con hijos(as) en edad preescolar, como el caso de Andrés y Fernando, se concentra en las mañanas y en las noches, para ellos es muy importante estar a la hora en que sus hijos(as) se despiertan, cambiar pañales, brindarles el alimento en el desayuno, bañarlos y jugar con ellos(as). El caso de Lorenzo y Ernesto cambia por el tema de la escuela, sin embargo, tratan de estar presentes en los requerimientos de sus hijas antes de empezar su jornada escolar. En la noche, todos los padres coinciden en permanecer en casa para estar al tanto de las peticiones de los(as) hijos(as). En los más pequeños, temas como dar el biberón, bañarlos y vestirlos para su descanso, y las infaltables levantadas a media noche a socorrer su llanto, son los quehaceres más comunes. Para los padres que tienen hijos(as) de mayor edad, la tarea se concentra en estar al tanto del cumplimiento de las tareas escolares y de un horario establecido para ir a la cama.

En los otros horarios, cuando los padres trabajan, los menores están en la escuela o están a cargo de familiares mujeres o nanas.

Las tías de mi esposo son las que nos ayudan a cuidar a mi hijo, ahorita por ejemplo él está con ellas, son como sus nanas, por decirlo así. Porque es un trato que hicimos de que ellas nos iban a ayudar para que nosotros pudiéramos seguir produciendo (Andrés).
En la mañana mi mamá limpia la casa, yo llevo a la niña a la escuela, ellas la recogen. Como yo llego más tarde, ellas le ayudan con la tarea, yo llego y se la reviso. Cuando yo estoy pues me la paso con ella viendo la tele, o le corto papel craft para que se ponga a pintar, cosas así (Lorenzo).
La que cuida a mi hija es la señora que siempre nos ha ayudado, que hace 10 años está en la casa; es nuestra empleada doméstica y ahí vive desde hace 10 años. Ella pasó a ser la nana y los fines de semana ella se va a su pueblo y viene su hermana, es la que se queda el fin de semana, pero siempre está una nana de todos modos (Fernando).

La ayuda en el cuidado de los menores siempre está a cargo de redes femeninas. En el caso de Ernesto, sus hijas también están bajo la asistencia de una empleada doméstica, que está al tanto de la alimentación y de la limpieza de la casa. En este sentido, por más que estamos tratando con arreglos familiares de varones gais en los que no está implícita una mujer de manera directa, las figuras femeninas tienen una presencia a la hora colaborar en los cuidados de los(as) hijos(as). Según el caso, la asistencia femenina tiene más centralidad en unos que en otros: en el caso de Lorenzo, su madre y su hermana son "las madres" de su pequeña, él lo declaró así, pues velan por ella la mayor parte del día; mientras que en otros casos la presencia es más accesoria y se limita a algunos horarios.

La asistencia en el cuidado de los pequeños por parte de familiares pone en evidencia un tema que tal vez es característico de las relaciones familiares en Latinoamérica, especialmente en México: las redes de apoyo. En el contexto regional las ayudas por parte de familiares, sobre todo de las abuelas, son un refugio fundamental para el cuidado de los menores. Por otra parte, la ayuda de nanas o de señoras que realizan el trabajo doméstico es un recurso al que pueden acceder solo ciertos sectores sociales. De acuerdo con Figueroa y Flores 
(2012), existe una relación directa entre cuidados y clase social, que además es complementada por el género, que interviene en la relación entre personas cuidadas y cuidadoras. Esta operación interviene en la división del trabajo en hogar y, particularmente, en las prácticas de paternidad de los participantes"

Pero vale la pena preguntarse ¿Qué actividades realizan estas mujeres? ¿Será que ejecutan las actividades más engorrosas mientras los padres están presentes en las más agradables? García y de Oliveira (2004) anotan que cuando se distingue entre el cuidado físico (alimentación y aseo) y la recreación, la presencia masculina se concentra más en este último aspecto. Lo mismo argumentan Bonino (2000) y Wainerman (2003) al afirmar que los padres tienden a implicarse más en las actividades agradables con sus hijos(as), pero sin modificar significativamente su implicación en las rutinas de la crianza ni en el resto del trabajo doméstico, porque son actividades consideradas como típicamente femeninas.

De acuerdo con los testimonios de los padres entrevistados, no se puede afirmar de manera certera que ellos estén de un lado u otro, aunque los casos son distintos según el padre. En el caso de Lorenzo, su asistencia en el cuidado de la niña está más relacionada con los espacios recreativos, las demás labores las realiza su madre y su hermana. Por su parte, Andrés y Fernando atienden funciones de alimentación y aseo de sus hijos(as), sin involucrarse mucho en la limpieza del hogar, pues tienen empleadas domésticas que les ayudan todos los días o unos días a la semana. En cuanto a Ernesto, la situación es un poco distinta porque sus hijas ya son adolescentes, sin embargo, también cuentan con la ayuda de una señora.

Por lo tanto, las redes femeninas son una ayuda fundamental para el cuidado de los(as) hijos(as) de los entrevistados. Otros estudios, como los de Mena (2009; 2013) sobre padres solteros, también llegaron a la misma conclusión; según la autora, la existencia, construcción y funcionamiento de las redes de apoyo condicionan el nivel de participación de los padres en el trabajo familiar, y en el cuidado y crianza de sus hijos(as), a la vez que son una fuente de bienestar para las familias ${ }^{12}$. Para algunos autores (Bonino 2000; Wainerman 2003; García y de Oliveira 2004; Mena 2009; 2013) esta situación muestra que las percepciones de los varones aún se encuentran muy apegadas a roles y estereotipos de género tradicionales, es decir, sigue reinando una devaluación de lo femenino (Echeverría 2013). Sin embargo, el caso de los padres entrevistados matiza esta idea, porque aunque relegan ciertas labores a las mujeres que les ayudan, al mismo tiempo se están involucrando en tareas que regularmente son tildadas de femeninas.

En definitiva, las dimensiones del tiempo dedicado a los hijos y de su cuidado en los arreglos familiares de los entrevistados no presentan grandes diferencias respecto a los resultados de otros estudios que involucran a familias heterosexuales de sectores medios. No obstante, existen particularidades como la participación de los padres en tareas del cuidado de los(as) pequeños(as), asunto

Il Al respecto, Gutmann (1997) subraya que la clase establece diferencias en los hábitos relacionados con las responsabilidades paternas, ya que los miembros de estratos más altos todavía pueden darse el lujo de contratar a otras personas para cuidar a sus hijos.

12 Según Mena (2013), la presencia y funcionamiento de estas redes de apoyo se hacen posibles gracias a los afectos que son reforzados por los lazos de parentesco. 
que en otros tipos de familia tienen un fuerte arraigo femenino. De igual manera, es importante señalar que estas dos dimensiones son cambiantes según la edad de los(as) hijos(as).

\section{Lo habitual y lo original en medio de la autoridad, los valores y la socialización de género de los menores}

Luego de revelar las dimensiones del tiempo y el cuidado descritos por los entrevistados, en los próximos renglones se detalla otra relación: ahora entre la autoridad -un asunto que también resulta ser central en el ejercicio paterno-, los valores inculcados a los menores y la socialización de género impartida por los padres. La selección de las dos últimas obedece a la búsqueda de nuevos referentes formativos que, según algunos estudios sobre maternidades o paternidades no heterosexuales (Donoso 2002; Haces 2006), ponen en escena algunos padres, aún más cuando son personas comprometidas políticamente con la diversidad sexual.

La autoridad es uno de los componentes principales con los que se ha asociado la paternidad. Pero, según Torres (2004), este mando que reviste la figura paterna genera conflictos en la relación con los(as) hijos(as), porque en ella recae la principal responsabilidad de los varones ${ }^{13}$. Sin embargo, los arreglos familiares han venido cambiando, sobre todo en contextos urbanos, no solo porque las madres estén más inmersas en la potestad del hogar, sino también porque los varones están dispuestos a entablar otro tipo de relaciones con los(as) hijos(as) que no se limiten al mando sobre ellos(as). Esto no quiere decir que no exista una disciplina sobre los menores, sino que se crean nuevas formas de construir relaciones padre/ hijo(a) que coordinan la potestad y los sentimientos sobre ellos(as).

Para conservar la figura de autoridad se acudía a actitudes como la distancia entre el padre y los(as) hijos(as), estar poco tiempo con ellos y asumir una frialdad sentimental. Ahora, las disposiciones de los padres han dejado atrás este apartamiento físico y afectivo para incorporar otras pautas de autoridad. Lo anterior tiene una resonancia directa en el tiempo dedicado a los menores y en las labores de cuidado, temas tratados renglones atrás.

A continuación algunos fragmentos de los testimonios de los padres cuando se les preguntó sobre el tema de la autoridad y castigo sobre los(as) hijos(as):

\footnotetext{
No tengo muchos problemas de disciplina, porque yo soy de la gente que se responsabiliza de lo que hace, y yo soy de: "no vas a hacer esto, entonces no hagas esto, y no pidas esto y punto", y no le estoy pegando, nada más no le doy las cosas y ya (Lorenzo).
}

Entonces él dice: "no, no me agarres, yo puedo agarrarme solo", y se le dice, "no, hijo, te tengo que agarrar porque si frena tu papá sales volando y no es correcto. O sea, lo

13 Sobre el tema, Figueroa (2011b) se pregunta ¿es posible la democracia en la familia?, argumentando que la figura de autoridad representada generalmente por el padre tiene el riesgo de caer en el autoritarismo y en el ejercicio unilateral de poder, asuntos que contradicen las nociones más difundidas de democracia. 
más que puedo hacer es, te dejo estar aquí, te agarras de acá y yo te voy agarrando”. Y eso ha sido un proceso de negociación, de mucha conversación (Andrés).

Ernesto habla sobre los pactos con sus hijas, que ya están adolecentes, para la hora de llegada de las salidas con sus amigos y novios:

\begin{abstract}
Yo le dije "a ver, si yo digo a las 7, vas a asumir ese límite tú, o vas a hacer lo que yo diga. Pon tu límite tú y cúmplelo". "Papá, a las ocho estoy en la casa", 8:05, 8:10 ya está en la casa. No es que yo diga a las 7 o a las 8 , ella pone su límite y cumple su límite (Ernesto).
\end{abstract}

Las nuevas actitudes frente a la disciplina y control sobre los(as) hijos(as) se sustentan básicamente en el diálogo y la negociación; el castigo físico no tiene entrada en este tipo de acuerdos familiares. Los padres entrevistados acuden a estrategias basadas en la autogestión y el reconocimiento de los límites por parte de los(as) propios(as) hijos(as) por medio de la conversación y del ejemplo. Dentro de las medidas llevadas a cabo por los varones se encuentran: las explicaciones detalladas a los menores sobre las consecuencias de los actos que están cometiendo o que quieren realizar, los diálogos en extenso sobre las reglas que se van a concertar entre padre e hijo(a) y el fomento a la responsabilidad sobre sí mismo(a).

El hecho de que se promueva la conciliación en las relaciones de autoridad con los descendientes no implica una desaparición de los castigos, sino que ya no son por medio de golpes u actos ofensivos. Los correctivos a los que acuden los padres entrevistados están básicamente relacionados con la privación de cosas o de actividades que les agradan a los menores. Para dos de los padres (Andrés y Fernando), las experiencias de castigo no han tenido mucha relevancia, pues sus hijos(as) están muy pequeños todavía. En el caso de Lorenzo, que su hija tiene seis años, la situación cambia debido a los actos de rebeldía que ha enfrentado con la pequeña, situaciones en las cuales no la deja ver la televisión, no le presta la computadora o no le permite realizar otras actividades como pintar en pliegos de papel por toda la casa. Ernesto afronta de manera muy distinta el tema de los castigos, pues sus hijas superan los 14 años de edad, con ellas puede entablar conversaciones con mayores niveles de discernimiento en las que se busca reconocer los errores y poner límites por su propia cuenta.

Entrelíneas se puede confirmar la plasticidad de los acuerdos de autoridad y disciplina de los entrevistados, que ya no acuden a lineamientos radicales para pretender un orden en el seno del hogar, sino que invitan a sus descendientes a ser partícipes de los acuerdos y así pretender una mejor convivencia ${ }^{14}$. Su actitud frente a la autoridad se asemeja a lo señalado por otros estudios (Nava 1996; Torres 2002; García y Oliveira 2004; Mena 2009) que apuntaron una mayor cercanía, presencia, comprensión, participación, comunicación y compromiso

14 Hay que tener en cuenta que lo afirmado por los entrevistados es un discurso construido en las entrevistas en el que los acuerdos de autoridad con sus hijos(as) parecen ser consistentes y coherentes, un tema en el que generalmente existen conflictos. 
de los padres con sus hijos(as), que a la vez indicaron la preferencia por la comunicación y el diálogo frente al regaño y al castigo.

De manera general, la orientación sexual de los padres entrevistados no tiene una implicación directa en la gestión de la disciplina y la autoridad sobre sus hijos(as). Sus testimonios permiten revelar que las maneras de asumir el mando sobre los menores no es distinto a lo revelado por los estudios sobre la paternidad en general. Así entonces, parece que las diferencias que se pueden encontrar entre las formas de asumir la autoridad no se precisan por medio de la orientación sexual de los padres, sino por otras categorías como la edad, el nivel educativo o el sector social de los involucrados, como lo han señalado otros estudios (Jiménez 2003; Salguero 2006; Rojas 2008).

Por ende, la emergencia de nuevas estrategias para gestionar la autoridad en la práctica paterna permite explorar las transformaciones del rol paterno contemporáneo, sobre todo en sectores medios urbanos, que básicamente intenta difuminar las jerarquías en la estructura familiar fomentando relaciones más igualitarias, asuntos que, como lo indica Figueroa (2011b), problematizan y reinventan ejercicios paternos más democráticos en los entornos familiares ${ }^{15}$. Aunque se debe aclarar que la autoridad no desaparece sino que se transforma, tomando nuevos formas para ejercerla, entre ellas, el diálogo y la comunicación.

Estas nuevas formas de asumir la autoridad están en sintonía con los valores que los padres pretenden inculcarles a los(as) hijos(as). Al ser el diálogo el principal instrumento de mediación, se genera mayor intimidad y confianza con los menores (Echeverría 2012), además que se fomenta otro tipo de principios rectores en sus vidas. Dentro de las negociaciones en torno a la disciplina subyacen fundamentalmente la responsabilidad y la autonomía, por lo que las maneras en las que los padres negocian la autoridad con los menores traen implícitas una serie de valoraciones, que son precisamente los principios a los cuales les están apostando en la crianza de sus descendientes.

Los valores inculcados a los(as) hijos(as) resultan ser otra categoría importante para rastrear en la práctica paterna, porque por medio de esta se pueden rastrear las posturas que toman los padres respecto a la educación de sus hijos(as), más ahora que el ejercicio de la paternidad se ha involucrado en otros espacios y actividades de la crianza de los menores. Según Nudler y Romaniuk (2005), estamos en un período de cambios en las subjetividades de hijos e hijas criados en familias innovadoras respecto del modelo tradicional, principalmente apostando a una democratización de los vínculos intergeneracionales $(2005,279)$. Estos cambios no repercuten solo en los(as) hijos(as), también en los padres que requieren nuevas herramientas simbólicas que les ayuden a establecer otros criterios para sobrellevar la relación familiar; entre esos mecanismos se encuentran los valores que traten de enseñarse.

15 Para complementar, el autor sugiere una serie de dimensiones para negociar en las relaciones familiares, principalmente entre padre e hijo(as), entre estas se encuentran la disciplina y la autoridad. Sobre la primera sostiene que existe un problema debido a que es regularmente el adulto el que se asume como disciplinador del menor de edad. Respecto a la segunda, argumenta que también se debería reconocer al hijo como autoridad, considerarlo como una persona con una historia específica y que seguramente por su especificidad puede generar aprendizajes sobre el padre (Figueroa 2011b, 87). 
En este sentido, más que a la autoridad, la paternidad está volcada a la educación, la comunicación, el conocimiento y a la confianza con los(as) hijos(as) (Alatorre y Luna 2000, 253). Algunos fragmentos sobre el tema:

Yo estoy tratando de encaminarlas a una libertad con responsabilidad; no tienen los parámetros clásicos del "debe ser asî". Aquí hay oportunidad de: ¿Tú cómo crees que debe ser? ¿Cómo dicen los demás que debe ser? Tomemos una decisión, ¿qué vas a hacer? (...) Les digo: yo lo que necesito es que ustedes sean unas personas que se puedan manejar por sí mismas, sean autosuficientes, y sean responsables de sus actos; con eso creo que es más que suficiente (Ernesto).

Lo más importante de ser papá es tener que apoyar a mi nena en lo que ella va a querer en su momento, a tomar sus decisiones y darle herramientas para que ella sea libre de tomar sus propias decisiones, que no tenga que tomarlas yo por ella, que ella sea la que las tome. (...) también generar una persona responsable, que asuma la responsabilidad de sus actos, porque mi nena, aun cuando tiene 5 años, es responsable de lo que hace, si ella tira algo, ella es responsable de arreglarlo (Lorenzo).

Para mí es importante que mi hijo vaya creándose criterios para decidir lo que quiere en la vida y ser autónomo; eso es algo muy importante (Andrés).

Para los padres entrevistados, la responsabilidad, la libertad y ser autosuficiente son las estimaciones que más tienen peso en la crianza de sus hijos(as). Ninguna de estas conlleva una figura autoritaria del padre, sino todo lo contrario, un padre cómplice de las decisiones y de los actos de los menores. A la vez, se está dibujando un padre acompañante de la libertad de los(as) hijos(as), siendo un guía en el camino y no un rector de los rumbos que tome la vida de sus hijos(as), por eso es tan importante la insistencia de los padres en formar personas independientes. Otro de los valores referidos por los padres fue el respeto por su hija, según Fernando, es un componente fundamental dentro de la crianza, porque le permite hacer un ejercicio de conciencia sobre su paternidad.

La actitud de los padres entrevistados respecto a la libertad y el respeto por las decisiones de los(as) hijos(as) es acorde a lo señalado por otros estudios. En palabras de Montesinos (2004), los padres están intentando establecer relaciones familiares libres de normas que provocaban distanciamiento afectivo y así forjar individuos autónomos y crear un ambiente familiar basado en la afectividad y el respeto (2004, 198). Idea que también es sustentada por Mena (2009), al comprobar que los padres están preocupados por conformar estructuras más democráticas y de mayor comunicación dentro de sus familias.

Inculcar principios morales, sin decir que religiosos, es sin duda una de las tareas en las cuales los padres están más abocados. Por esta razón no se puede asegurar que la transmisión de valores solo hace parte de los modelos de paternidad contemporáneos, porque en estructuras familiares de antaño, los varones también participaban en la inculcación de principios rectores sobre sus hijos(as). Lo que ha cambiado son los valores que se infunden y los modos de hacerlo. Este tipo de transformaciones, como lo argumenta Olavarría (2000), permiten observar la lucha que se produce entre los miembros de la familia por conseguir mayores espacios de libertad y autonomía así como de relaciones más igualitarias. 
La insistencia de crear nuevos marcos de autoridad y de valores es un fenómeno que intenta introducirse en el ejercicio paterno, principalmente, de varones jóvenes, de sectores socioeconómicos medios y altos, y de niveles educativos altos ${ }^{16}$. En este sentido, la orientación sexual de los padres no tiene una repercusión directa en el fomento de la responsabilidad y la libertad sobre sus hijos(as), tampoco en el respeto de sus decisiones. Lo que sí se puede sospechar en los discursos de los varones entrevistados es su preocupación por ser padres innovadores, que siempre están buscando estar "in" en cuanto a los modelos de padres contemporáneos. Son personas que están al tanto de las primicias sobre pautas de crianza y educación de los(as) hijos(as) por medio de internet, revistas especializadas o por consejos de amigos(as) ${ }^{17}$.

Un punto vinculado con los valores que sí es distinto de los padres entrevistados es el fomento a la tolerancia y al respeto de la diversidad ${ }^{18}$.

\begin{abstract}
Yo lo que pretendo es que mi hijo vea todo tipo de personas, para que así le conste que existen formas diversas de vivir, porque así como ha asistido a las marchas gais y a espectáculos gais o a reuniones donde ve gente bastante estrafalaria, también ha ido a peregrinaciones religiosas con sus tías, yo dejo que lo lleven. (...) Yo busco que crezca libre, que crezca teniendo referentes de todo, de todo en la vida, desde sabores, desde olores, desde gustos, desde gustos de todo tipo, musicales..., que sé yo, para que partir de ahí él vaya creándose criterios para decidir lo que quiere en la vida (Andrés).
\end{abstract}

Andrés no fue el único que hizo alusión al tema de la diversidad y la tolerancia, los demás padres insistieron que en el trato con sus hijos(as) se esfuerzan por inculcarles el respeto por las demás personas. El fomento a este tipo de valores no se dirige en exclusiva al tema de la diversidad sexual, también incumbe otro tipo de asuntos como el origen étnico, la situación de pobreza, personas con diversidad funcional, entre otros. Los padres en sus testimonios declaran su compromiso para que sus hijos(as) no reciban ninguna enseñanza que pudiera convertirse en una práctica discriminatoria, eso tal vez, porque como lo señalaron algunos, no quieren que sus descendientes promuevan actividades de las que ellos en algún momento fueron víctimas ${ }^{19}$.

El proceder de los padres para incentivar una educación abierta a la tolerancia y a la diferencia es variado. Algunas actividades son: llevar a los menores a eventos o reuniones a las que asisten personas de diversa índole en cuanto a su sexualidad o corporalidad y leerles cuentos con enfoques abiertos a la pluralidad (este material era compartido por los padres en internet). El fomento al respeto, la tolerancia y la solidaridad es importante para los padres porque se convierte en el granito de

$16 \mathrm{Al}$ respecto, los estudios de Jiménez (2003), García y De Oliveira (2004) y Rojas (2008) argumentan que estas categorías sociodemográficas son fundamentales para comprender las prácticas de paternidad de los varones involucrados en sus investigaciones.

17 En repetidas ocasiones, Andrés y Fernando publicaban en sus redes sociales de internet artículos o revistas sobre paternidad y pautas de crianza.

18 En el mismo sentido, Donoso (2002) en su estudio con madres lesbianas en España sostiene que son mujeres que están intentando criar hijos(as) mucho más abiertos a la diferencia, que no sean homofóbicos y con todos los medios para superar el estigma asociado a su condición familiar.

19 En un artículo de prensa, Andrés insiste en que su hijo está creciendo en un hogar donde se le está preparando para defenderse y para ser muy respetuoso con las demás personas. 
arena con el cual ellos están colaborando para transformar los regímenes de discriminación promovidos actualmente. Ellos esperan que sus hijos(as) sean partícipes de la transformación a nuevos enfoques de convivencia que involucren a cualquier persona sin ningún reparo.

Así entonces, la paternidad de los varones entrevistados le está apostando a la trasmisión de nuevos valores que no han sido consignados en otros estudios sobre paternidad en la región. La preocupación por inculcar principios hacia el respeto y la tolerancia se puede sumar a las demás estimaciones que los padres en la actualidad le están instruyendo a sus hijos(as); sin embargo, parece que existe un acento especial de las paternidades no heterosexuales, que son precisamente las que llevan a cuesta una historia de discriminación en su contra ${ }^{20}$.

Otro de los ejercicios impulsados por los padres entrevistados es su recelo por establecer una socialización de género con tintes heteronormativos sobre los menores.

\begin{abstract}
Yo no creo en lo masculino y lo femenino; a mi nena, si quiere pinturas de nena, se las compro, si quiere una patineta, se la compro, o sea, yo no creo en esas cosas. (...) Yo no le impongo cosas que son típicas del estereotipo como perforarle los oídos, de pequeña ella eligió como a los 4 años que ella quería los aretes, sí fue por influencia de la abuela o algo, pero ella fue la que me lo pidió: "quiero mis aretes", pero antes yo no dejé que le pusieran aretes (Lorenzo).
\end{abstract}

Aquí intentamos que eso de los roles de género establecidos no lo vivamos (Andrés).

El intento de establecer nuevos referentes en la socialización de los menores es una tarea que tal vez puede resultar difícil. Por una parte, se requiere de recursos simbólicos que desvirtúen el fuerte arraigo heterosexual de la cultura, lo que implica un ejercicio de desestimación de normas culturales y simultáneamente la creación de nuevos marcos de sentido. Por otra parte, a la hora de la realización de los ejercicios de socialización innovadores se reciben señalamientos de otros espacios como la familia extensa o la escuela. Por estos motivos, los padres necesitan tener perseverancia para no dejar en ciernes la tarea que se propusieron.

Algunos estudios sostienen que en el ejercicio de la paternidad se construyen y naturalizan las relaciones entre los géneros (Alatorre y Luna 2000; Mena 2009; 2013; Salguero y Pérez 2008), y al mismo tiempo, los(as) hijos(as) reciben los discursos sobre las formas aceptadas de ser hombre o de ser mujer. Por eso, las apuestas protagonizadas por los padres entrevistados por estructurar otras formas de socialización tienen una justificación política y familiar, pues son posturas que intentan transformar regímenes de poder y discriminación sustentados en un orden de género arbitrario. Además, como lo declaran Barker y Verani (2008), la niñez y la adolescencia son momentos cruciales para llegarles a los chicos y jóvenes con mensajes alternativos sobre género y masculinidad.

Así entonces, parece que los padres abordados están dando pie a la creación de nuevos imaginarios y nuevas formas de crianza que integren otros valores y otras prácticas a la hora de educar a los(as) hijos(as). De acuerdo con lo anterior, Cebotarev

Sobre el tema, Haces (2006) en su estudio sobre homofamilias en México, también descubre que existe una preocupación por educar y formar en un ambiente de respeto y tolerancia. 
(2003) manifiesta que en las prácticas de paternidad los padres tienen libertad de introducir conscientemente prácticas parentales con estilos que faciliten y promuevan el desarrollo de nuevas generaciones $(2003,78)$. Tal vez esta novedad en los modelos de crianza sea promovido también en arreglos familiares heterosexuales, no obstante, el peso que tienen la orientación sexual de los padres entrevistados y su activismo político para decidir sobre estos asuntos son de vital importancia para comprender las estrategias que tratan de llevar a cabo, pues uno de sus objetivos es intervenir en los marcos culturales que a veces se tornan cerrados y discriminatorios, y de los cuales en algún momento fueron víctimas. Algunos dilemas descritos por los entrevistados son: dar explicaciones sobre su paternidad en espacios públicos y a desconocidos, y la selección de la escuela.

Estas modificaciones en los modelos de crianza dan cuenta de que la educación moral o en valores es una vía para desarticular algunos imaginarios sociales. En otras palabras, son transformaciones que se convierten en un intento por combatir un orden heteronormativo que, entre otras cosas, se encarga de instaurar mayores expectativas de obligación, compromiso y responsabilidad hacia los(as) hijos(as) y hacia la sociedad en las parejas del mismo sexo que a las de tipo heterosexual (Weeks, Heaphy y Donovan 1999). Al respecto, Judith Butler (2006), al preguntarse si el parentesco es de antemano heterosexual, argumenta que en el trasfondo de los debates sobre la reproducción por parte de parejas del mismo sexo existe una premura por ver a los(as) hijos(as) como un sitio extremo para la transferencia y la reproducción de cultura, y de hecho es así, o por lo menos eso lo comprueban los testimonios de los varones entrevistados, que al ser conscientes de las consecuencias discriminatorias que sustentan algunos marcos culturales, procuran transformarlos al intervenir de manera directa en la educación de los(as) hijos(as) ${ }^{21}$. De todas maneras, hay que tener en cuenta que la transferencia de la cultura no se agota en la crianza de los(as) hijos(as), pues existen otros mecanismos en los cuales también se lleva a cabo esta tarea.

Las apuestas por nuevas pautas en la socialización primaria de los menores no se limita a una figura de género exclusiva, es decir, que porque son dos padres entonces el(la) hijo(a) solo tenga referentes masculinos. Todo lo contrario, son menores que también tienen referentes femeninos, y que a través de ellos también reciben ciertos códigos de crianza a los cuales los padres se están atreviendo. De acuerdo con las palabras de los entrevistados, las abuelas o las tías que cuidan a sus pequeños(as), o en otros casos la nana, son los referentes femeninos con los cuales están creciendo sus descendientes. No obstante, algunas veces ellas son las que estropean las pautas novedosas de crianza establecidas por los padres. A continuación un apartado en el que Lorenzo declara sus esfuerzos por que su familia no imponga algunos principios en los que él no está de acuerdo: “... yo lo intento evitar. En todas las familias, digo. Mi mamá y mi hermana por más que lo

21 Figueroa y Flores (2012) sostienen que las formas de socialización alternativas que reciben los(as) hijos(as) de ciertos grupos sociales pueden consolidarse en sus propios grupos familiares y de esa manera convertirse en una vía de transformación social $(2012,53)$. Idea que también es recalcada por Boscán (2008) al señalar que la construcción de nuevas masculinidades requiere de un trabajo que debe comenzarse desde la infancia, noción que, según Carabí y Segarra (2000), es reconocida principalmente por su carácter antisexista y antihomófobo. 
intentan, tienen una vena heteronormativa muy cabrona, pero yo intento que no se transmita".

En definitiva, los padres entrevistados están inmersos en actitudes poco tradicionales a la hora de encarar su ejercicio paterno, principalmente, en lo que concierne al manejo de la autoridad, la transmisión de valores y a una socialización de género. Aunque existan ciertos obstáculos para desplegar a todas sus anchas sus modelos de crianza, como la familia extensa o la escuela, los padres entrevistados contaron con los recursos necesarios para seguir adelante con su proyecto.

\section{Reflexiones finales}

Los testimonios de los entrevistados permiten presumir a grandes rasgos que a la hora de hablar de paternidad (cuando estamos haciendo referencia a padres que residen con sus descendientes) concurren componentes transversales que se enmarcan en la relación de una persona mayor con una criatura que necesita ser protegida y socializada. En este sentido, como lo indica Haces (2006), la reflexión en torno a la paternidad gay implica irremediablemente repensar la paternidad en general. Asuntos como el cuidado, la socialización y la formación moral son aspectos que no presentan peculiaridades de fondo entre la paternidad de los padres abordados y las conclusiones de los estudios revisados. En su interior, los arreglos parentales tienen similitudes a la hora de asumir la crianza de los(as) hijos(as), pero las cosas cambian cuando los padres deben voltear la cara al exterior; es allí donde emergen las singularidades de las formaciones parentales diversas. En este sentido, el dualismo público/privado resulta ser fundamental para equiparar o distinguir la paternidad de varones gais con otros arreglos parentales.

La premisa fundamental de la que partió la investigación hacía alusión a la reproducción de marcos heteronormativos por parte de los padres gais debido a que era el único antecedente que se tenía para asumir una relación paterna. A lo largo del texto se pudo comprobar que no hay estrictamente una réplica de este precepto, más bien lo que existe es una apuesta por involucrar nuevos modelos de paternidad en los que también están inmersos algunos padres heterosexuales, principalmente de sectores sociales medios y con un nivel educativo alto.

No se puede olvidar que la paternidad de los entrevistados, pese a que está marcada profundamente por su orientación sexual, también está mediada por otras categorías como la edad, la clase, el nivel educativo, el carácter urbano de su residencia y su activismo político en defensa de la diversidad sexual. La edad, la pertenencia a los sectores medios y la educación acumulada de los padres les permite gozar de recursos materiales y simbólicos para asumir algunos traspiés con sus hijos(as), entre ellos, la promoción de pautas de crianza acordes con la llamada por algunos estudios "paternidad contemporánea". Por otra parte, el contexto de México D.F. está a su favor, pues el anonimato que brinda la ciudad y el fomento a la diversidad sexual promovido por el gobierno local son plataformas que favorecen las formaciones familiares diversas. Por último, el activismo político de los padres resultó ser un antecedente substancial para la agencia de su paternidad desde el proceso de decisión hasta la actualidad; es un 
referente que les ha brindado estrategias para blindar su conformación familiar de las nociones discriminatorias en su contra.

En términos metodológicos, los límites de campo no fueron un obstáculo categórico para el desarrollo de la investigación. A pesar que solo se contó con el consentimiento de cuatro padres y de la aproximación a su experiencia únicamente por medio de la entrevista, se pudo realizar un análisis eficiente sobre las categorías propuestas en el estudio. La circunstancia de escasez de participantes y de materiales de información provocó la búsqueda de otros medios que menguaran está condición, uno de ellos fue rastrear materiales que me revelaran otros ángulos de la relación paterna como fotografías, videos, blogs y artículos de prensa en los que algunos de ellos eran sus personajes centrales. Otra fórmula fue otorgarle más protagonismo y profundidad a cada caso. Así entonces, la renuencia de los padres gais no debe ser catalogada como un condicionante trascendental para el acercamiento a este tema de estudio, más bien se deben explorar otros caminos para llegar a su experiencia.

Para terminar, el presente estudio comprueba que la relación entre el trabajo y la paternidad tienen una alta incidencia en el ejercicio de la paternidad, asunto que ha sido sostenido por otros estudios y que además es un apunte que demuestra que la proveeduría económica sigue siendo un asunto fundamental para los padres, aun siendo gais y estando emparejados con otro varón. De igual forma, parece que en las situaciones familiares en las que no está involucrada de manera directa una mujer, como es el caso de las familias homoparentales de varones, sigue habiendo una feminización de ciertos quehaceres del cuidado y de las labores domésticas, debido principalmente al poco auspicio y adiestramiento que reciben los hombres desde pequeños para estas actividades.

Con relación a la autoridad, los valores trasmitidos a los(as) hijos(as) y la socialización no sexista, se confirma que pese a que las paternidades de hombres gais, como las de los cuatro entrevistados, estén en sintonía con las transformaciones que ha venido sustentando la práctica paterna en las últimas décadas (cercanía con los(as) hijos(as) y la democratización de las relaciones familiares) aún es necesario reforzar otros contenidos relacionados con la homofobia. Tanto así, que no solo blinda su arreglo familiar de posibles señalamientos externos, en una especie de eventualidad imaginada, sino que desde su interior intenta establecer ejercicios de crianza que deslegitimen prácticas discriminatorias contra la diversidad sexual y contra otras categorías susceptibles de segregación.

\section{Referencias bibliográficas}

Alatorre, Javier y Rafael Luna. «Significados y prácticas de la paternidad en la Ciudad de México». En Paternidades en América Latina, editado por Norma Fuller, 241-275. Lima: Pontificia Universidad Católica del Perú, Fondo Editorial, 2000.

Barker, Gary y Fabio Verani. La participación del hombre como padre en la región de Latinoamérica y el Caribe: una revisión de literatura crítica con consideraciones para políticas. Brasil: Promundo-Save the Children, 2008.

Bonino, Luis. Los varones hacia la paridad en lo doméstico-discursos sociales y prácticas masculinas. 2000. http://www.uv.es/ dones/temasinteres/paridad. pdf (último acceso: mayo de 2014). 
Bonino, Luis. «Las nuevas paternidades». Cuadernos de trabajo social, Vol. 16, 2003: 171-182.

Boscán, Antonio. «Las nuevas masculinidades positivas». Utopía y praxis latinoamericana, $\mathrm{n}^{\circ}$ 13, 2008: 93-103.

Butler, Judith. Deshacer el género. Barcelona: Paidós, 2006.

Carabí, Angels y Marta Segarra. Nuevas masculinidades. Barcelona: Icaria Editorial, 2000.

Cebotarev, Nora. «Familia, socialización y nueva paternidad». Revista Latinoamericana de Ciencias Sociales, Niñez y Juventud, Vol. 1, n² 2, 2003: 53-78.

Donoso, Silvia. «Epílogo. La familia lésbica». En Gestión familiar de la homosexualidad, de Gilbert Herdt y Bruce Koff, 171-213. Barcelona: Bellaterra, 2002.

Echeverría, Genoveva. «Ser padre fuera de la familia: subjetividad y vínculos de varones padres que ya no viven con sus hijos». La ventana, $\mathrm{n}^{\circ}$ 36, 2012: 292-334.

Echeverría, Genoveva. "Vulnerabilidad en los varones mexicanos: fisuras y aperturas en las subjetivaciones masculina». En Los hombres en México: veredas recorridas y por andar. Una mirada a los estudios de género de los hombres, las masculinidades, coordinado por Juan Carlos Ramírez y Juan Carlos Cervantes, 91-109. Guadalajara: Universidad de Guadalajara, CUCEA, AMEGH-A.C, 2013.

Figueroa, Juan. «Paternidad, mortalidad y salud: ¿Es posible combinar estos términos?» En Estudios sobre varones y masculinidades para la generación de políticas públicas y acciones transformadoras, de IV Coloquio Internacional de Estudios sobre Varones y Masculinidades Montevideo, 71-78. Uruguay: Universidad de la República, $2011 a$.

Figueroa, Juan. “¿Es posible la democracia en la familia? Algunas reflexiones a partir del ejercicio de la paternidad». En Agentes de desarrollo local para la democratización familiar. Experiencias en el oriente del Estado de México, coordinado por Beatriz Scmuker, 80-89. México D.F.: Instituto Mora, 2011 b.

Figueroa, Juan y Natalia Flores. «Prácticas de cuidado y modelos emergentes en las relaciones de género. La experiencia de algunos varones mexicanos». La ventana, $\mathrm{n}^{\circ} 35,2012:$ 7-57.

Figueroa, Juan y Josefina Franzoni. «Del hombre proveedor al hombre emocional: construyendo nuevos significados de la masculinidad entre varones mexicanos». En Masculinidades y políticas públicas. Involucrando hombres en la equidad de género, coordinado por Francisco Aguayo y Michelle Sadler, 6483. Santiago de Chile: Universidad de Chile, 2011.

Fuller, Norma. «Significados y prácticas de paternidad entre varones urbanos del Perú». En Paternidades en América Latina, editado por Norma Fuller, 35-90. Lima: Pontificia Universidad Católica del Perú, 2000.

García, Brígida y Orlandina de Oliveira. «El ejercicio de la paternidad en el México urbano». En Imágenes de la familia en el cambio de siglo, coordinado por Marina Ariza y Orlandina de Oliveira, 283-317. México D.F.: Instituto de Investigaciones Sociales -UNAM, 2004.

Gutmann, Matthew. «Machos que no tienen ni madre. Paternidad y masculinidad en la Ciudad de México». La ventana, $\mathrm{n}^{\circ}$ 7, 1997: 120-165.

Gutmann, Matthew. Ser hombre de verdad en la ciudad de México: ni macho ni mandilón. México D.F.: El Colegio de México, 2000. 
Haces, María. ¿Maternidad lésbica, paternidad gay? Del autoreconocimiento homoerótico al ejercicio paternal. Una aproximación antropológica a las homofamilias. México D.F.: CIESAS, tesis de doctorado en Antropología, 2006.

Herzberg, Yuri. Familias queer en la sociedad de la Ciudad de México. Construcción de espacios público-privados. México D.F.: UNAM, tesis de maestría en Estudios Políticos y Sociales, 2005.

Jiménez, Lucero. Dando voz a los varones. Sexualidad, reproducción y paternidad de algunos mexicanos. México D.F.: Centro Regional de Investigaciones Multidisciplinarias, UNAM, 2003.

Laguna, Oscar. Arreglos parentales de los varones gay en la Ciudad de México: ¿desestabilización o continuidad?. México D.F.: Universidad Autónoma Metropolitana Xochimilco, tesis de doctorado en Ciencias Sociales, 2013.

Libson, Micaela. «Parentalidades gays y lesbianas: varones y mujeres en familias no heteronormativas». La ventana, $\mathrm{n}^{\circ}$ 35, 2012: 292-321.

Mena, Paulina. Ser padres solteros en la Ciudad de México y el área metropolitana. México D.F.: PIEM/Colegio de México, tesis de maestría en Estudios de Género, 2009.

Mena, Paulina. Cuando los varones se quedan con sus hijos. La experiencia de las familias de padres solteros en Querétaro. México D.F.: CIESAS, tesis de doctorado en Antropología, 2013.

Mena, Paulina y Laura Torres. «Prácticas paternas en divorciados, viudos y abandonados». En Los hombres en México: veredas recorridas y por andar. Una mirada a los estudios de género de los hombres, las masculinidades, coordinado por Juan Carlos Ramírez y José Carlos Cervantes, 71-89. Guadalajara: Universidad de Guadalajara, CUCEA, AMEGH-A.C, 2013.

Montesinos, Rafael. «La nueva paternidad: expresión de la transformación masculina». Polis: Investigación y Análisis Sociopolítico y Psicosocial, Vol. 2, $\mathrm{n}^{\circ}$ 4, 2004: 197-220.

Nava, Regina. Los hombres como padres en el Distrito Federal a principios de los noventa. México D.F.: UNAM, tesis de maestría en Sociología, 1996.

Nudler, Alicia y Susana Romaniuk. «Prácticas y subjetividades parentales: transformaciones e inercias». La Ventana, $\mathrm{n}^{\circ}$ 22, 2005: 269-285.

Olavarría, José. «Ser padre en Santiago de Chile». En Paternidades en América Latina, editado por Norma Fuller, 129-174. Lima: Pontificia Universidad Católica del Perú, Fondo Editorial, 2000.

Rojas, Olga. Paternidad y vida familiar en la Ciudad de México: un estudio del desempeño masculino en los procesos reproductivos y en la vida doméstica. México D.F.: El Colegio de México, 2008.

Salguero, María. «Significado y vivencia de la paternidad en algunos varones de los sectores socioeconómicos medios en la Ciudad de México». En Ser padres, esposos e hijos: prácticas y valoraciones de varones mexicanos, coordinado por Juan Guillermo Figueroa, Lucero Jiménez y Olivia Tena, 57-94. México D.F.: El Colegio de México, 2006.

Salguero, María y Gilberto Pérez. La paternidad en los varones: una búsqueda de identidad en un terreno desconocido. Algunos dilemas, conflictos y tensiones. 2008. http://www.estudiosmasculinidades.buap.mx/num4/varones.htm (último acceso: mayo de 2014). 
Secretaría de Desarrollo Social (Sedesol), Consejo Nacional de Población (Conapo), Instituto Nacional de Estadística y Geografía (Inegi), Instituto de Investigaciones Sociales de la UNAM y el Colegio de México. Diferentes tipos de familias mexicanas. 2013.http://www.uniradioinforma.com/noticias/graficosespeciales/254649/diferentes-tipos-de-familias-mexicanas.html (último acceso: junio 2014).

Torres, Laura. Ejercicio de la paternidad en la crianza de hijos e hijas. México D.F.: UNAM, Tesis de doctorado en sociología, Facultad de Ciencias Políticas y Sociales, 2002.

Torres, Laura. «La paternidad: una mirada retrospectiva». Revista de Ciencias Sociales, Vol. 3, 2004: 47-58.

Viveros, Mara. «Paternidades y masculinidades en el contexto colombiano contemporáneo, perspectivas teóricas y analíticas». En Paternidades en América Latina, editado por Norma Fuller, 90-128. Lima: Pontificia Universidad Católica del Perú, 2000.

Wainerman, Catalina. Familia, trabajo y género: un mundo de nuevas relaciones. Buenos Aires: UNICEF/Fondo de Cultura Económica, 2003.

Weeks, Jeffrey, Brian Heaphy y Catherine Donovan. «Partners by Choice: Equality, Power and Commitment in Non-Heterosexual Relationships». In Sociology of Family, editado por Allan Johnson, ll1-128. Oxford: Blackwell, 1999.

\section{Entrevistas}

Andrés, 45 años, paternidad en pareja. Entrevista realizada por Sebastián Giraldo el 05 y 12 de junio de 2013 en México D.F.

Ernesto, 57 años, paternidad en soltería. Entrevista realizada por Sebastián Giraldo el 22 y 29 de mayo del 2013 en México D.F.

Fernando, 47 años, paternidad en pareja. Entrevista realizada por Sebastián Giraldo el 30 de mayo y 6 de junio del 2013 en México D.F.

Lorenzo, 43 años, paternidad en soltería. Entrevista realizada por Sebastián Giraldo el 4 de junio del 2013 en México D.F. 